\title{
Factorization model for distributions of quarks in hadrons
}

\author{
B. I. Ermolaev ${ }^{1, a}$, M. Greco ${ }^{2}$, S. I. Troyan ${ }^{3}$ \\ ${ }^{1}$ Ioffe Physico-Technical Institute, 194021 St. Petersburg, Russia \\ 2 Department of Mathematics and Physics and INFN, University Roma Tre, Rome, Italy \\ ${ }^{3}$ St. Petersburg Institute of Nuclear Physics, 188300 Gatchina, Russia
}

Received: 5 March 2015 / Accepted: 27 May 2015 / Published online: 4 July 2015

(C) The Author(s) 2015. This article is published with open access at Springerlink.com

\begin{abstract}
We consider distributions of unpolarized (polarized) quarks in unpolarized (polarized) hadrons. Our approach is based on QCD factorization. We begin with a study of the basic factorization for the parton-hadron scattering amplitudes in the forward kinematics and suggest a model for non-perturbative contributions to such amplitudes. This model is based on this simple observation: after emitting an active quark by the initial hadron, the remaining set of quarks and gluons becomes unstable, so a description of this colored state can approximately be done in terms of resonances, which leads to expressions of the Breit-Wigner type. Then we reduce these formulas to obtain explicit expressions for the quark-hadron scattering amplitudes and quark distributions in $K_{T}$ - and collinear factorizations.
\end{abstract}

\section{Introduction}

QCD factorization, i.e. separation of perturbative and nonperturbative QCD contributions, proved to be an efficient instrument for describing hadron reaction at high energies. Being first applied to processes in the hard kinematics in the form of collinear factorization [1-13], it was soon extended to cover the forward kinematic region, with DGLAP [14-17] used to account for the perturbative contributions. Then, in order to be able to use BFKL [18-20], a new kind of factorization, $K_{T}$-factorization was suggested in Ref. [21,22]. These kinds of factorization are usually illustrated by identical pictures. For instance, factorization of the DIS hadronic tensor $W_{\mu \nu}$ is conventionally depicted by the construction in Fig. 1 both in collinear and in $K_{T}$-factorizations, where the upper, perturbative blob and the lower, non-perturbative blob are connected by two-parton state. The upper blob in Fig. 1 is calculated with regular perturbative means. On the contrary, the lower blob is conventionally introduced from purely phenomenological considerations. Collinear and

a e-mail: boris.ermolaev@cern.ch
$K_{T}$-factorizations operate with different parametrizations for momentum $k$ of the connecting partons and, as a result, they are described by different formulas. Collinear factorization assumes that

$k=\beta p$,

while $K_{T}$-factorization allows for the transverse momentum in addition:

$k=\beta p+k_{\perp}$,

accounting therefore for one longitudinal and two transverse components of $k$. However, as a matter of fact, $k$ has four components: two of them are longitudinal and the other two are transverse. Accounting for the missing longitudinal component $\alpha$ (for the definition of $\alpha$ see Eq. (3)) drove us to suggesting a new, more general factorization, which we called in Ref. [23,24] basic factorization. In contrast to $K_{T}$ - and collinear factorizations, the analytic expressions in basic factorization can be obtained from the graphs of the type of the one in Fig. 1 with applying the standard Feynman rules.

It is worth recalling briefly our derivation of basic factorization; for details see Ref. $[23,24]$. Let us consider the Compton scattering amplitude off a hadron in the forward kinematics. It is depicted in Fig. 2.

The blob in Fig. 2 denotes an ensemble of perturbative and non-perturbative contributions. This blob can be expanded into an infinite series of terms; each of them is represented by two blobs connected with $n$ parton lines, $n=2,3, \ldots$ Considering only the simplest, two-parton state, we arrive at the graph similar to the one in the r.h.s. of Fig. 1 but without the $s$-cut and with both blobs accommodating perturbative and non-perturbative contributions at the same time. The integration of the convolution in Fig. 1 over momentum $k$ now runs over the whole phase space and it is expected to lead to a finite result. However, the propagators of the connecting partons become singular at $k^{2}=0$ (we neglect quark masses). Besides, the upper blob may contain IR-sensitive perturbative 


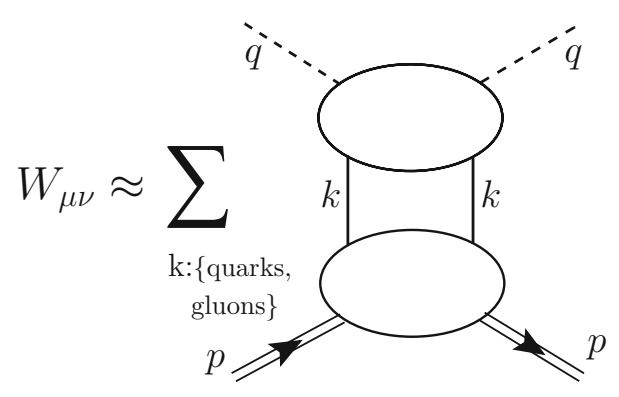

Fig. 1 Conventional illustration of QCD factorization. The $s$-cut of the graph is implied

contributions $\sim \ln ^{n}\left(2 p k / k^{2}\right)$ (with $\left.n=1,2, \ldots\right)$. In addition, it yields the factor $2 q k / k^{2}$, when unpolarized gluon ladders are included into the consideration. The only way to kill such an IR singularity is to assume that the lowest, non-perturbative blob should tend to zero fast enough when $k^{2} \rightarrow 0$. Doing so and repeating a similar procedure to regulate the UV singularity, we bring the convolution in Fig. 1 to agreement with the factorization concept: perturbative and non-perturbative contributions are located in different blobs. This is a new form of QCD factorization which we name basic factorization.

We demonstrated in Ref. [23,24] that basic factorization can be reduced step-by-step first to $K_{T}$ - and then to collinear factorizations. In Ref. [23,24] we began with considering basic factorization for Compton scattering amplitudes in the forward kinematics, where integration over momentum $k$ of the connecting partons in Fig. 1 runs over the whole phase space. Confronting the two obvious facts that, on the one hand, the integration over $k$ should yield a finite result and that, on the other hand, the perturbative part in Fig. 1 (the upper, perturbative blob and propagators of the connecting partons) is divergent in both the infra-red (IR) and ultra-violet (UV) regions allowed us to impose integrability restrictions on the lowest blob, which are necessary for the convolution in Fig. 1 to be finite. The obtained restrictions led us to theoretical constraints on the fits for the parton distributions to the DIS structure functions in collinear and $K_{T}$-factorizations. In particular, we predicted the general form of the fits in $K_{T^{-}}$ factorization and excluded the factors $x^{-a}$ from the fits in both $K_{T^{-}}$and collinear factorizations.

Another interesting object, where factorization is used, is the distribution of partons in hadrons. In the present paper we examine their properties in the IR and UV regions and suggest a simple resonance model for the non-perturbative contributions to the parton distributions. Our argumentation in favor of this model is as follows: after emitting an active quark by a hadron, the remains of the hadron, i.e. a set of quarks and gluons, acquires a color and therefore it becomes unstable. So, this colored state can be described in terms of resonances. We begin with considering amplitudes of the quark-hadron (QHA) and gluon-
Fig. 2 Amplitude for forward Compton scattering off a hadron target

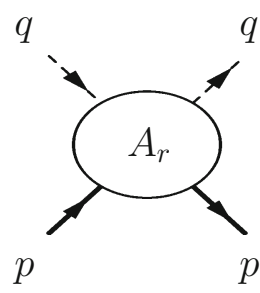

hadron (GHA) scattering in the forward kinematics. The optical theorem relates such amplitudes to the parton distributions. Throughout the paper we use the standard Sudakov parametrization [25] for the momentum $k$ of the connecting partons:

$k=-\alpha q^{\prime}+\beta p^{\prime}+k_{\perp}$,

where momenta $q^{\prime}$ and $p^{\prime}$ are massless, $p^{\prime 2} \approx q^{\prime 2} \approx 0$, and they are made of the hadron momentum $p$ and the parton momentum $q$ :

$p^{\prime}=p+x_{2} q, \quad q^{\prime}=q+x_{1} p$,

where $x_{2}=-p^{2} / w \equiv-M^{2} / w, x_{1}=-q^{2} / w$, with $w=2 p q \approx 2 p^{\prime} q^{\prime}$. In these terms

$2 p k=w\left(-\alpha-x_{2} \beta\right), \quad 2 q k=w\left(\beta-x_{1} \alpha\right)$,

$k^{2}=-w \alpha \beta-k_{\perp}^{2}$.

In Sect. 2 we introduce the quark-hadron scattering amplitudes in the forward kinematics and examine their IR and UV behavior. In Sect. 3 we consider separately the unpolarized and spin-dependent quark-hadron amplitudes in basic factorization and suggest a model for non-perturbative contributions to the amplitudes. This model involves a spinor structure accompanied by invariant amplitudes $T^{(U)}$ and $T^{(S)}$. In Sect. 3 we specify the spinor structure of the non-perturbative contributions to the amplitudes and parton distributions. In Sect. 4 we show how basic factorization for the quark-hadron amplitudes and quark distributions in hadrons can be reduced to $K_{T}$ - and collinear factorizations. In Sect. 5 we focus on a model for the invariant amplitudes $T^{(U)}$ and $T^{(S)}$. The model is based on a description of $T^{(U)}$ and $T^{(S)}$ in a quasi-resonant way and through the optical theorem it easily leads to nonperturbative contributions to the parton distributions, with expressions of the Breit-Wigner kind both in the basic and in the $K_{T}$-factorizations. Finally, Sect. 6 is for concluding remarks.

\section{Quark-hadron amplitudes}

In the factorization approach, the quark-hadron amplitudes (QHA) $A_{\mathrm{q}}$ are expressed through convolutions of perturbative amplitudes $A^{\text {(pert) }}$ and non-perturbative amplitudes $T$ as shown in Fig. 3. 


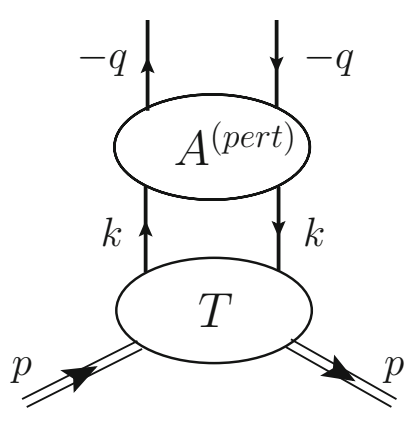

Fig. 3 Factorization of the quark-hadron amplitude

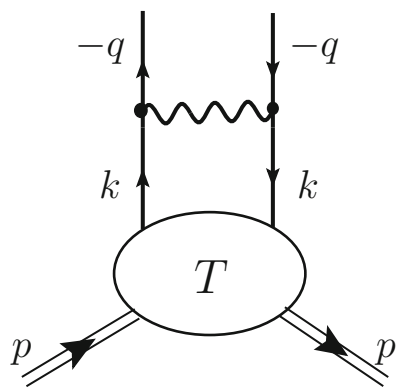

Fig. 4 Born approximation for the factorized quark-hadron amplitude

In the Born approximation $A^{\text {(pert) }}$ is depicted in Fig. 4 as a one-rung ladder. Adding more ladder rungs together with the inclusion of non-ladder graphs and resumming all such graphs convert the Born amplitude into $A^{\text {(pert) }}$. In the present paper we do not consider mixing of quark and gluon ladder rungs, i.e. we consider the graphs where the vertical quark lines go from the bottom to the top without breaking.

We begin with consideration of the quark-hadron amplitudes $A_{\mathrm{q}}$ in basic factorization, studying the simplest case depicted in Fig. 4, where the perturbative contributions are accounted for in the Born approximation and we denote such distributions $B_{\mathrm{q}}$. In basic factorization one can use the standard Feynman rules to write down the analytic expression corresponding to the graphs in Figs. 3 and 4. Doing so, we obtain

$B_{\mathrm{q}}=-l 4 \pi \alpha_{s} C_{F} \int \frac{\mathrm{d}^{4} k}{(2 \pi)^{4}} \frac{\bar{u}(q) \gamma_{\mu} \hat{k} \hat{T}_{\mathrm{q}}(k, p) \hat{k} \gamma_{\nu} u(q)}{k^{2} k^{2}(q+k)^{2}} d_{\mu \nu}$,

where we have used the standard notation $C_{F}=\left(N^{2}-\right.$ $1) /(2 N)=4 / 3$ and $\alpha_{s}$ is the QCD coupling. In Eq. (6) $\hat{T}_{\mathrm{q}}$ corresponds to the lowest blob in Fig. 3. It is an altogether non-perturbative object. Throughout the paper we will call it the primary quark-hadron amplitude. ${ }^{1}$ Choosing the Feynman gauge, where $d_{\mu \nu}=g_{\mu \nu}$, for the virtual gluon and the

${ }^{1}$ In Ref. [26,27] non-perturbative contributions to parton distributions in the context of collinear factorization were called intrinsic contributions.
Sudakov parametrization (3) for the quark momentum $k$, we rewrite Eq. (6) as follows:

$$
B_{\mathrm{q}}=-l \frac{\alpha_{s} C_{F}}{8 \pi^{3}} w \int \mathrm{d} \alpha \mathrm{d} \beta \mathrm{d}^{2} k_{\perp} \frac{\bar{u}(q) \gamma_{\mu} \hat{k} \hat{T}_{\mathrm{q}}(k, p) \hat{k} \gamma_{\mu} u(q)}{k^{2} k^{2}(q+k)^{2}} .
$$

Throughout the paper, for the sake of simplicity, we will treat the external quarks with momentum $q$ as on-shell ones, though our reasoning remains valid also when they are offshell. Introducing the density matrix

$\hat{\rho}(p)(q)=\frac{1}{2}\left(\hat{q}+m_{\mathrm{q}}\right)\left(1-\gamma_{5} \hat{S}_{\mathrm{q}}\right)$,

with $q, m_{\mathrm{q}}$ and $S_{\mathrm{q}}$ being the quark momentum, mass, and spin, respectively, we bring Eq. (7) to the following form:

$B_{\mathrm{q}} \approx-l \frac{\alpha_{s} C_{F}}{8 \pi^{3}} w \int \mathrm{d} \alpha \mathrm{d} \beta \mathrm{d}^{2} k_{\perp} \frac{\operatorname{Tr}\left[\hat{\rho}(p)(q) \gamma_{\mu} \hat{k} \hat{T}_{\mathrm{q}}(k, p) \hat{k} \gamma_{\mu}\right]}{k^{2} k^{2}(q+k)^{2}}$.

We stress that the replacement of Eq. (7) by Eq. (9) is not necessary for us but it allows us to carry out a more detailed consideration of $A_{\mathrm{q}}^{B}$. In particular, we can consider separately the spin-dependent, $B_{\mathrm{q}}^{\text {(spin) }}$, and independent, $B_{\mathrm{q}}^{\text {(unpol) }}$, quark-hadron amplitudes in a simple way:

$$
\begin{aligned}
B_{\mathrm{q}}^{(\text {unpol })}= & -l \frac{\alpha_{s} C_{F}}{8 \pi^{3}} w \int \mathrm{d} \alpha \mathrm{d} \beta \mathrm{d}^{2} k_{\perp} \\
& \times \frac{2(q k) \operatorname{Tr}\left[\hat{k} \hat{T}_{\mathrm{q}}^{(\text {unpol })}\right]-k^{2} \operatorname{Tr}\left[\hat{q} \hat{T}_{\mathrm{q}}^{\text {(unpol })}\right]}{k^{2} k^{2}(q+k)^{2}}, \\
B_{\mathrm{q}}^{\text {(spin) }}= & \frac{\alpha_{s} C_{F}}{8 \pi^{3}} m_{\mathrm{q}} w \int \mathrm{d} \alpha \mathrm{d} \beta \mathrm{d}^{2} k_{\perp} \\
& \times \frac{2\left(S_{\mathrm{q}} k\right) \operatorname{Tr}\left[\gamma_{5} \hat{k} \hat{T}_{\mathrm{q}}^{\text {(spin) }}\right]-k^{2} \operatorname{Tr}\left[\gamma_{5} \hat{S}_{\mathrm{q}} \hat{T}_{\mathrm{q}}^{\text {(spin) }}\right]}{k^{2} k^{2}(q+k)^{2}} .
\end{aligned}
$$

In Eqs. (10) and (11) we have replaced the general primary amplitude $\hat{T}_{\mathrm{q}}$ by the more specific amplitudes $\hat{T}_{\mathrm{q}}^{\text {(unpol) }}$, $\hat{T}_{\mathrm{q}}^{\text {(spin) }}$. In Eq. (10) we have neglected a contribution $\sim m$ in $\hat{\rho}(q)$ compared to the contribution $\sim \hat{q}$. Integrations in Eqs. (10) and (11) run over the whole phase space and this is supposed to yield finite results. However, there can be singularities in the integrands and they should be regulated. Regulating them with introducing various cut-offs would be unphysical, so the only way out is to impose appropriate constraints on the primary quark-hadron amplitudes, $\hat{T}_{\mathrm{q}}^{\text {(unpol) }}, \hat{T}_{\mathrm{q}}^{\text {(spin) }}$, so that we kill the singularities. When the perturbative amplitude $A^{\text {(pert) }}$ is calculated in the Born approximation, the only possible singularities in Eqs. (10) and (11) are IR singularities at $k^{2}=0$ and UV singularities which we relate to integrations over $\alpha$. However, when $A^{\text {(pert) }}$ is beyond the Born approximation, there appear other 
kinds of singularities, called in Ref. [28] rapidity divergences. Below we consider the handling of these singularities in the framework of basic factorization.

\subsection{Rapidity divergences of QHA}

Rapidity divergences were investigated first in Ref. [28] and then in Ref. [29,30] in the context of $K_{T}$-factorization. Detailed investigation of this problem can be found in Ref. [31]. In the lowest order of perturbative QCD, the rapidity divergences come from the graphs in Fig. 5 (and symmetrical graphs as well), where the radiative corrections calculated in the first-loop approximation are convoluted with the unintegrated parton distribution $\widetilde{\Phi}$. Let us stress that $\widetilde{\Phi}$ accumulates both perturbative and non-perturbative corrections. When such convolutions are considered in $K_{T}$-factorization, each of the graphs in Fig. 5 acquires logarithmic divergences arising from integration over the momentum $l_{+}$(with $\left.l_{+}=\left(l_{0}+l_{z}\right) / \sqrt{2}\right)$. They are called rapidity divergences and they can be got rid of as shown in Ref. [28] when the Feynman gauge is used for the gluon propagators and in Ref. [29,30] for the case of the light-cone gauge. In Refs. [28-30] the rapidity divergences are cured by redefining $\widetilde{\Phi}$.

Now let us study this situation in basic factorization. To this end we consider a contribution of the graph in Fig. 6 to the quark-hadron amplitude in basic factorization. We recall that there are no cuts in Fig. 6 and the blob $T$ accumulates non-perturbative contributions only.

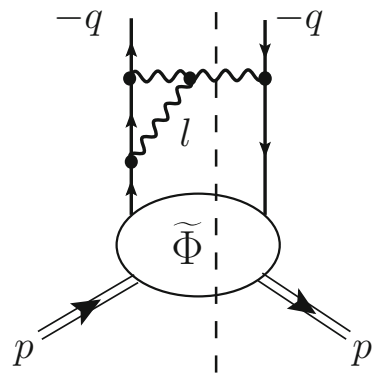

(a)

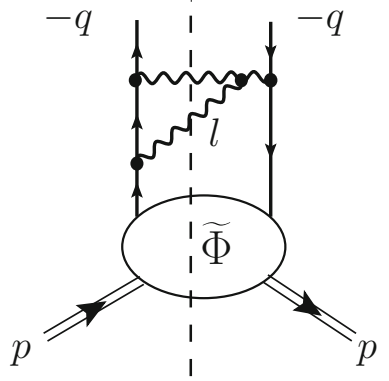

(b)
Fig. 5 Graphs contributing to rapidity divergences in unintegrated parton distributions. The dashed lines denote cuts

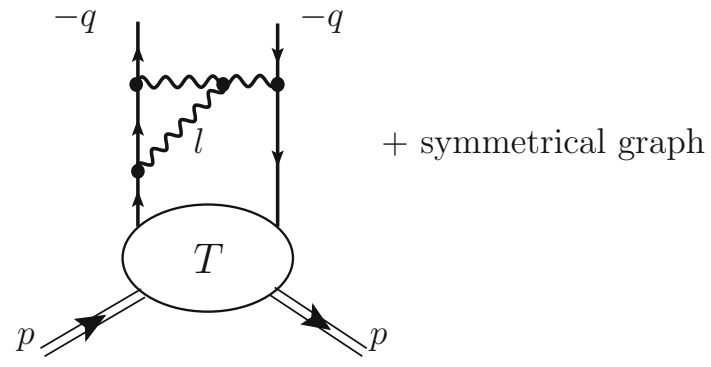

Fig. 6 Graph contributing to quark-hadron amplitude
One of the remarkable features here is that analytic expressions in basic factorization can be obtained by applying standard Feynman rules to the involved graphs. A second important point is that one is free to use any gauge for perturbative QCD calculations ${ }^{2}$ in basic factorization, whereas the blob $T$ in Fig. 6 is altogether non-perturbative and therefore it is insensitive to the choice of the gauge. Applying the Feynman rules to the graph in Fig. 6 and integrating over the loop momentum $l$, we immediately conclude that this integration yields a logarithmic UV-divergent contribution, which, being complemented by a similar contribution from the symmetrical graph and self-energy graphs, in a conventional way leads to renormalization of the gluon-quark couplings. After absorption of such divergent contributions by the couplings, we obtain a renormalized amplitude which is free of divergences. Then applying the optical theorem to this construction, we arrive at the parton distributions and they are also free of divergences. Obviously, the same treatment can be applied to other UV divergences coming from the perturbative component $A^{\text {(pert) }}$ in higher loops: all of them can be absorbed by renormalizations. Now we focus on the divergences resulting from integration of the convolutions in Eqs. (10) and (11), where the perturbative amplitudes $A^{\text {(pert) }}$ are in the Born approximation.

\subsection{IR and UV stability of QHA}

First of all, let us note that the denominators in Eqs. (10) and (11) can become singular in the infra-red (IR) region, where $k^{2} \sim 0$. In the case of purely perturbative QCD, IR singularities are conventionally regulated by introducing IR cut-offs. In our case there is no physical reason for that at all, so we are left with the only way to kill these singularities: The primary quark-hadron amplitudes $\hat{T}_{\mathrm{q}}$ should become small at small $k^{2}$ :

$\hat{T}_{\mathrm{q}}^{\text {(unpol) }}, \hat{T}_{\mathrm{q}}^{\text {(spin) }} \sim\left(k^{2}\right)^{1+\eta}$,

when $k^{2} \rightarrow 0$. Now let us consider the ultra-violet (UV) stability of the convolutions in Eqs. (10) and (11). The integration over $\alpha$ in Eqs. (10) and (11) runs from $-\infty$ to $\infty$, so at large $|\alpha|$ the integrands should decrease fast enough to guarantee UV stability. First of all we focus on the integration over $\alpha$ in Eq. (10). Taking into consideration that each factor in the denominator of Eq. (10) is $\sim \alpha$ makes the denominator $\sim \alpha^{3}$. The term $2 q k$ in the numerator depends on $\alpha$ because $2 q k=w\left(\beta-x_{1} \alpha\right)$ and the factors $k^{2}$ and $\hat{k}$ are $\sim \alpha$, which leads to

$\frac{2 q k \hat{k}}{k^{2} k^{2}(q+k)^{2}} \sim \frac{\alpha^{2}}{\alpha^{3}}$.

\footnotetext{
${ }^{2}$ For gauge invariance of basic factorization see Ref. [23,24].
} 
This divergence must be regulated by an appropriate decrease of $\hat{T}_{\mathrm{q}}^{\text {(unpol) }}$ at large $|\alpha|$. The IR stability condition in Eq. (12) states that $\hat{T}_{\mathrm{q}}^{\text {(unpol) }} \sim\left(k^{2}\right)^{1+\eta}$ at small $k^{2}$ but it can either disappear or be kept at large $|\alpha|$. Therefore we have two options:

(A) The factor $\left(k^{2}\right)^{1+\eta}$ survives at large $|\alpha|$.

(B) The factor $\left(k^{2}\right)^{1+\eta}$ disappears at large $|\alpha|$.

In the case (A), where IR and UV behaviors of $\hat{T}_{\mathrm{q}}^{\text {(unpol) }}$ are related, $\hat{T}_{\mathrm{q}}^{\text {(unpol) }}$ should behave at large $|\alpha|$ as follows:

$\hat{T}_{\mathrm{q}}^{\text {(unpol) }} \sim \alpha^{\eta-\chi}=\left(\alpha^{1+\eta}\right)\left[\alpha^{-1-\chi}\right]$,

with $\chi>\eta>0$.

The IR and UV behaviors of $\hat{T}_{\mathrm{q}}^{\text {(unpol) }}$ are disconnected in the case (B). It converts Eq. (14) into

$\hat{T}_{\mathrm{q}}^{\text {(unpol) }} \sim \alpha^{-\chi}$.

The first factor in Eq. (14) corresponds to the term $\left(k^{2}\right)^{1+\eta}$, while a contribution generating the asymptotic factor in the squared brackets has to be specified. We will do this in Sect. 5 . Now let us consider the spin-dependent amplitudes. In order to guarantee their IR stability, the primary spin-dependent amplitude $\hat{T}_{\mathrm{q}}^{\text {(spin) }}$ should also be $\sim\left(k^{2}\right)^{1+\eta}$ at small $k^{2}$ but the situation with its UV stability is more involved than in the unpolarized case. Indeed, the quark spin $S_{\mathrm{q}}$ can be either in the plane formed by $p$ and $q$, i.e. $S_{\mathrm{q}}=S_{\mathrm{q}}^{\|}$, or in the transverse space, where $S_{\mathrm{q}}=S_{\mathrm{q}}^{\perp}$. Depending on it, there are the longitudinal spin-dependent amplitude, $B_{\mathrm{q}}^{\|}$and the transverse one, $B_{\mathrm{q}}^{\perp}$. Now let us consider the term $2 m_{\mathrm{q}} S_{\mathrm{q}} k$ in Eq. (11) for different orientations of the quark spin. When the spin is longitudinal,

$2 m_{\mathrm{q}} S_{\mathrm{q}} k=2 m_{\mathrm{q}} S_{\mathrm{q}}^{\|} k=w\left(\beta-x_{1} \alpha\right)$

and $\hat{k}$ in the trace $\operatorname{Tr}\left[\hat{k} \hat{T}_{\mathrm{q}}\right]$ is also $\sim \alpha$. In contrast when the spin is transverse,

$2 m_{\mathrm{q}} S_{\mathrm{q}} k=-2 m_{\mathrm{q}}\left(\vec{S}_{\mathrm{q}}^{\perp} \vec{k}_{\perp}\right)$,

and therefore $S_{\mathrm{q}}^{\perp} k$ does not depend on $\alpha$. Then this $\vec{k}_{\perp}$ should be accompanied by another $\vec{k}_{\perp}$ from the trace in order to get a non-zero result at integration over the azimuthal angle. The first term in the numerator of Eq. (11) does not depend on $\alpha$, while the second term is $\sim \alpha$. It means that, with $\hat{T}_{\mathrm{q}}^{\| l}$ dropped, the explicit $\alpha$-dependence of $A_{\mathrm{q}}^{\|}$at large $|\alpha|$ coincides with the one in Eq. (13):

$\frac{S_{\mathrm{q}}^{\|} k \hat{k}}{k^{2} k^{2}(q+k)^{2}} \sim \frac{\alpha^{2}}{\alpha^{3}}$

and

$\frac{S_{\mathrm{q}}^{\perp} k \hat{k}}{k^{2} k^{2}(q+k)^{2}} \sim \frac{\alpha}{\alpha^{3}}$.
It follows from Eq. (18) that the $\alpha$-dependence of the amplitude $\hat{T}_{\mathrm{q}}^{\|}$at large $|\alpha|$ is identical to the one of $\hat{T}_{\mathrm{q}}^{\text {(unpol) }}$ :

$\hat{T}_{\mathrm{q}}^{\|} \sim \hat{T}_{\mathrm{q}}^{(\text {unpol })} \sim\left(\alpha^{1+\eta}\right)\left[\alpha^{-1-\chi}\right]$

in the case $(\mathrm{A})$ and

$\hat{T}_{\mathrm{q}}^{\|} \sim \hat{T}_{\mathrm{q}}^{\text {(unpol) }} \sim\left(\alpha^{-\chi}\right)$

in the case (B). $\hat{T}_{\mathrm{q}}^{\perp}$ can decrease more slowly:

$\hat{T}_{\mathrm{q}}^{\perp} \sim\left(\alpha^{1+\eta}\right)\left[\alpha^{-\chi}\right]$

in the case $(\mathrm{A})$ and

$\hat{T}_{\mathrm{q}}^{\perp} \sim \alpha^{1-\chi}$

in the case (B). Equations (12) and (20-23) guarantee the integrability of the convolutions for the quark-hadron amplitudes in basic factorization. These integrability requirements can be used as general theoretical constraints on nonperturbative contributions to the amplitudes in basic factorization (see Ref. [23,24] for detail) and we will use them in the present paper. Each of Eqs. (20) and (22) consists of two factors. The first factor in these equations is universally generated by the term $\left(k^{2}\right)^{1+\eta}$, while contributions generating the factors in squared brackets will be specified in Sect. 5 .

\section{Modeling the spinor structure of $\hat{T}_{q}$}

Our next step is to simplify the traces in Eqs. (10) and (11). In order to do it, we have to specify the spinor structure of the primary QHA $\hat{T}_{\mathrm{q}}$. By definition, $\hat{T}_{\mathrm{q}}$ is altogether nonperturbative, so specifying its spinor structure can only be done on the basis of phenomenological considerations. However, any model expression for $\hat{T}_{\mathrm{q}}$ should respect the integrability conditions in Eqs. (12) and (14) and (20) and (22). There is the well-known expression for the density matrix of an elementary fermion:

$\hat{\rho}(p)=\frac{1}{2}(\hat{p}+M)\left(1-\gamma_{5} \hat{S}\right) \approx \frac{\hat{p}}{2}-\frac{1}{2}(\hat{p}+M) \gamma_{5} \hat{S}$,

where $M$ and $S$ are the fermion mass and spin. This expression leads us to approximate $\hat{T}_{\mathrm{q}}$ as follows:

$\hat{T}_{\mathrm{q}}=\hat{p} T_{\mathrm{q}}^{(U)}\left(k^{2}, 2 p k\right)-(\hat{p}+M) \gamma_{5} \hat{S} T_{\mathrm{q}}^{(S)}\left(k^{2}, 2 p k\right)$,

where $p, S$ are the hadron momentum and spin, respectively, and $T_{\mathrm{q}}^{(U)}, T_{\mathrm{q}}^{(S)}$ are scalar functions. Throughout the paper we will call them invariant quark-hadron amplitudes. Substituting $T_{\mathrm{q}}$ of Eq. (12) in Eqs. (10) and (11) and calculating the traces, we arrive at the following expressions: 


$$
\begin{aligned}
B_{\mathrm{q}}^{(\text {unpol })}= & -l \frac{1}{8 \pi^{3}} \int \mathrm{d} \alpha \mathrm{d} \beta \mathrm{d} k_{\perp}^{2}\left[-g^{2} C_{F} \frac{w}{\left[(q+k)^{2}+\imath \epsilon\right]}\right] \\
& \times\left(\frac{k_{\perp}^{2}}{k^{2} k^{2}}\right)\left(T_{\mathrm{q}}^{(U)}\left(k^{2}, 2 p k\right)\right) \\
= & -l \frac{1}{8 \pi^{3}} \int \mathrm{d} \alpha \mathrm{d} \beta \mathrm{d} k_{\perp}^{2} \widetilde{B}_{\mathrm{q}}^{(\text {unpol })}(q, k) \\
& \times\left(\frac{k_{\perp}^{2}}{k^{2} k^{2}}\right)\left(T_{\mathrm{q}}^{(U)}\left(k^{2}, 2 p k\right)\right),
\end{aligned}
$$

were we have denoted by $\widetilde{B}_{\mathrm{q}}^{\text {(unpol) }}$ the perturbative amplitude in the Born approximation for the forward annihilation of unpolarized quark-quark pair. We have neglected contributions $\sim x_{1,2}$ in the numerator of Eq. (26) and will do this in expressions for the spin-dependent amplitudes. These terms, if necessary, can easily be accounted for with a more accurate implementation of Eqs. (3)-(26). Let us consider the structure of the integrand in Eq. (26) in more detail. The amplitude in the last brackets is entirely non-perturbative. It is supposed to mimic a transition from hadrons to quarks. The fraction in the middle corresponds to convoluting the perturbative and non-perturbative amplitudes. The fraction in the first brackets corresponds to the perturbative amplitude for the forward scattering of quarks in the Born approximation. We explicitly wrote the factor $l \epsilon$ there to recall that this amplitude has an $s$-channel imaginary part. Doing similarly, we obtain an expression for the spin-dependent amplitudes:

$$
\begin{aligned}
B_{\mathrm{q}}^{\text {(spin) }}= & \iota \frac{g^{2} C_{F}}{16 \pi^{4}} 2 m_{\mathrm{q}} M w \int \mathrm{d} \alpha \mathrm{d} \beta \mathrm{d}^{2} k_{\perp} \\
& \times \frac{2\left(k S_{\mathrm{q}}\right)(k S)-k^{2}\left(S_{\mathrm{q}} S\right)}{k^{2} k^{2}\left[(q+k)^{2}+\imath \epsilon\right]} T_{\mathrm{q}}^{(S)} .
\end{aligned}
$$

Let us consider Eq. (27) for a different orientation of the hadron spin:

(i) The hadron spin $S$ is in the plane formed by momenta $p$ and $q$, so for this case we use the notation $S=S^{\|}$.

(ii) The hadron spin is transverse to this plane. We denote this case as $S=S^{\perp}$.

Amplitude $A_{\mathrm{q}}^{\|}$for the first case is given by the expression very close to the unpolarized amplitude:

$$
\begin{aligned}
B_{\mathrm{q}}^{(\|)}= & -l \frac{1}{16 \pi^{3}} \int \mathrm{d} \alpha \mathrm{d} \beta \mathrm{d} k_{\perp}^{2}\left[-g^{2} C_{F} \frac{2 m M\left(S_{\mathrm{q}}^{\|} S^{\|}\right)}{(q+k)^{2}+\imath \epsilon}\right] \\
& \times\left(\frac{k_{\perp}^{2}}{k^{2} k^{2}}\right) T_{\mathrm{q}}^{(\|)}\left(k^{2}, 2 p k\right) \\
= & -l \frac{1}{8 \pi^{4}} \int \mathrm{d} \alpha \mathrm{d} \beta \mathrm{d}^{2} k_{\perp} \widetilde{B}_{\mathrm{q}}^{(\|)}(q, k) \\
& \times\left(\frac{k_{\perp}^{2}}{k^{2} k^{2}}\right) T_{\mathrm{q}}^{(\|)}\left(k^{2}, 2 p k\right),
\end{aligned}
$$

whereas the transverse amplitude is given by a different expression:

$$
\begin{aligned}
B_{\mathrm{q}}^{(\perp)}= & -l \frac{1}{16 \pi^{3}} \int \mathrm{d} \alpha \mathrm{d} \beta \mathrm{d} k_{\perp}^{2}\left[-g^{2} C_{F} \frac{2 m M\left(S_{\mathrm{q}}^{\perp} S^{\perp}\right)}{(q+k)^{2}+\imath \epsilon}\right] \\
& \times\left(\frac{w \alpha \beta}{k^{2} k^{2}}\right) T_{\mathrm{q}}^{(\perp)}\left(k^{2}, 2 p k\right) \\
= & -l \frac{1}{8 \pi^{3}} \int \mathrm{d} \alpha \mathrm{d} \beta \mathrm{d} k_{\perp}^{2} \widetilde{B}_{\mathrm{q}}^{(\perp)}(q, k) \\
& \times\left(\frac{w \alpha \beta}{k^{2} k^{2}}\right) T_{\mathrm{q}}^{(\perp)}\left(k^{2}, 2 p k\right),
\end{aligned}
$$

with $\widetilde{B}_{\mathrm{q}}^{(\|)}, \widetilde{B}_{\mathrm{q}}^{(\perp)}$ being the perturbative spin-dependent Born amplitudes. Accounting for perturbative $\mathrm{QCD}$ radiative corrections converts the Born amplitudes $\widetilde{B}_{\mathrm{q}}^{(\text {unpol })}, \widetilde{B}_{\mathrm{q}}^{(\|)}, \widetilde{B}_{\mathrm{q}}^{(\perp)}$ in Eqs. (26), (28), and (29) into perturbative dimensionless amplitudes $\widetilde{A}_{\mathrm{q}}^{(\text {unpol })}, \widetilde{A}_{\mathrm{q}}^{(\|)}, \widetilde{A}_{\mathrm{q}}^{(\perp)}$, the other factors remaining unchanged:

$$
\begin{aligned}
A_{\mathrm{q}}^{(\text {unpol })}(p, q)= & -l \frac{1}{8 \pi^{3}} \int \mathrm{d} \beta \frac{\mathrm{d} k_{\perp}^{2}}{k^{2}} \mathrm{~d} \alpha \widetilde{A}_{\mathrm{q}}^{(\text {unpol })}(q, k) \\
& \times\left(\frac{k_{\perp}^{2}}{k^{2}}\right) T_{\mathrm{q}}^{(U)}\left(k^{2}, 2 p k\right), \\
A_{\mathrm{q}}^{(\|)}(p, q)= & -l \frac{1}{8 \pi^{3}} \int \mathrm{d} \beta \frac{\mathrm{d} k_{\perp}^{2}}{k^{2}} \mathrm{~d} \alpha \widetilde{A}_{\mathrm{q}}^{(\|)}(q, k) \\
& \times\left(\frac{k_{\perp}^{2}}{k^{2}}\right) T_{\mathrm{q}}^{(\|)}\left(k^{2}, 2 p k\right), \\
A_{\mathrm{q}}^{(\perp)}(p, q)= & -l \frac{1}{8 \pi^{3}} \int \mathrm{d} \beta \frac{\mathrm{d} k_{\perp}^{2}}{k^{2}} \mathrm{~d} \alpha \widetilde{A}_{\mathrm{q}}^{(\perp)}(q, k) \\
& \times\left(\frac{w \alpha \beta}{k^{2}}\right) T_{\mathrm{q}}^{(\perp)}\left(k^{2}, 2 p k\right) .
\end{aligned}
$$

Taking the $s$-imaginary part of Eq. (26), we arrive at the totally unintegrated, or fully unintegrated as stated in Ref. [32], distribution of unpolarized quarks in the hadron $D_{\mathrm{q}}^{\text {unpol B }}$ in the Born approximation:

$$
\begin{aligned}
D_{\mathrm{q}}^{(\text {unpol B) }}= & \frac{1}{8 \pi^{2}} \int \mathrm{d} \beta \frac{\mathrm{d} k_{\perp}^{2}}{k^{2}} \mathrm{~d} \alpha\left[g^{2} C_{F} \delta(\beta-x-z)\right] \\
& \times \frac{k_{\perp}^{2}}{k^{2}} \Psi_{\mathrm{q}}^{(1)}\left(k^{2}, 2 p k\right) \\
= & \frac{1}{8 \pi^{2}} \int \frac{\mathrm{d} \beta}{\beta} \frac{\mathrm{d} k_{\perp}^{2}}{k^{2}} \mathrm{~d} \alpha\left[g^{2} C_{F} \delta(1-x / \beta-z / \beta)\right] \\
& \times \frac{k_{\perp}^{2}}{k^{2}} \Psi_{\mathrm{q}}^{(1)}\left(k^{2}, 2 p k\right)
\end{aligned}
$$

where $x=-q^{2} / w, \quad z=k_{\perp}^{2} / w$, and $\Psi_{\mathrm{q}}^{(1)}$ is the primary quark distribution of unpolarized quarks in the hadron, $\Psi_{\mathrm{q}}^{(1)}=(1 / \pi) \Im T_{\mathrm{q}}^{(U)}$. This object is altogether nonperturbative. Applying the optical theorem to Eq. (30), we arrive at the parton distributions beyond the Born approximation: 


$$
\begin{aligned}
& D_{\mathrm{q}}^{(\text {unpol) }}\left(x, q^{2}\right) \\
& =\frac{1}{8 \pi^{2}} \int \frac{\mathrm{d} \beta}{\beta} \frac{\mathrm{d} k_{\perp}^{2}}{k^{2}} \mathrm{~d} \alpha \widetilde{D}_{\mathrm{q}}^{(\text {unpol })}\left(x / \beta, q^{2} / k^{2}\right) \mathrm{d} \beta\left(\frac{k_{\perp}^{2}}{k^{2}}\right) \\
& \quad \times \Psi_{\mathrm{q}}^{(1)}\left(k^{2}, w \alpha\right), \\
& D_{\mathrm{q}}^{(\|))}\left(x, q^{2}\right)=\frac{1}{8 \pi^{2}} \int \frac{\mathrm{d} \beta}{\beta} \frac{\mathrm{d} k_{\perp}^{2}}{k^{2}} \mathrm{~d} \alpha \widetilde{D}_{\mathrm{q}}^{(\|)}\left(x / \beta, q^{2} / k^{2}\right)\left(\frac{k_{\perp}^{2}}{k^{2}}\right) \\
& \quad \times \Psi_{\mathrm{q}}^{(\|)}\left(k^{2}, w \alpha\right), \\
& D_{\mathrm{q}}^{(\perp)}\left(x, q^{2}\right)=\frac{1}{8 \pi^{2}} \int \frac{\mathrm{d} \beta}{\beta} \frac{\mathrm{d} k_{\perp}^{2}}{k^{2}} \mathrm{~d} \alpha \widetilde{D}_{\mathrm{q}}^{(\perp)}\left(x / \beta, q^{2} / k^{2}\right)\left(\frac{w \alpha \beta}{k^{2}}\right) \\
& \quad \times \Psi_{\mathrm{q}}^{(\perp)}\left(k^{2}, w \alpha\right) .
\end{aligned}
$$

\section{Reduction of basic factorization to conventional factorizations}

The conventional forms of factorization are the collinear and the $k_{T}$-factorizations. In Ref. $[23,24]$ we described the reduction of basic factorization to $K_{T}$ - and collinear factorizations for the Compton scattering amplitudes and DIS structure functions without specifying the non-perturbative amplitudes $T_{\mathrm{q}}$. In this section we show that these results perfectly agree with our assumption in Eq. (25) concerning the structure of $T_{\mathrm{q}}$. We demonstrate that the parton distributions in both conventional factorizations can be obtained with stepby-step reductions of the expressions for $D_{\mathrm{q}}^{(\text {unpol })}, D_{\mathrm{q}}^{(\|)}, D_{\mathrm{q}}^{(\perp)}$ in basic factorization. These reductions are the same for both the parton-hadron amplitudes and the parton distributions, they are insensitive to the spin and stands when the quarks are replaced by gluons. Because of that we consider such reductions for a generic parton-hadron distribution $D$ in basic factorization and skip unessential factors:

$$
\begin{aligned}
D\left(x, q_{\perp}^{2}\right)= & \int \mathrm{d} \beta \frac{\mathrm{d} k_{\perp}^{2}}{k^{2}} \mathrm{~d} \alpha D^{(\text {pert })}\left(x / \beta, q_{\perp}^{2} / k^{2}\right)\left(\frac{k_{\perp}^{2}}{k^{2}}\right) \\
& \times \Psi\left(w \alpha, k^{2}\right),
\end{aligned}
$$

where $D^{\text {(pert) }}$ stands for a perturbative contribution and $\Psi$ is the altogether non-perturbative (we call it primary) partonhadron distribution. Actually, $\Psi\left(w \alpha, k^{2}\right)$ is the starting point for the perturbative evolution. Integration in Eq. (33) runs over the whole phase space. Let us note that in the literature very often are considered purely transverse $q: q^{2} \approx q_{\perp}^{2}$. Because of this we will use the notation $q_{\perp}^{2}$ instead of $q^{2}$ in what follows, though Eqs. (32) and (33) are also valid when $q^{2} \neq q_{\perp}^{2}$.

\subsection{Reduction to $k_{T}$-factorization}

In order to reduce Eq. (33) to $k_{T}$-factorization, we have to perform an integration with respect to $\alpha$. However, this inte- gration should not involve $D^{\text {(pert) }}$, which, strictly speaking, is impossible because $D^{\text {(pert) }}$ depends on $k^{2}$ and thereby it depends on $\alpha$ : $k^{2}=-w \alpha \beta-k_{\perp}^{2}$. The only way out is to assume that the main contributions to Eq. (33) come from the region where

$\alpha \ll \alpha_{\max }=k_{\perp}^{2} /(w \beta)$,

i.e. $k^{2} \approx-k_{\perp}^{2}$. Let us notice that approximating ladder partons virtualities $k^{2}$ by their transverse momenta is well known. It is used in all available evolution equations, including DGLAP and BFKL, and now it allows us to convert Eq. (33) into an expression for the unintegrated (transverse momentum dependent [33]) parton distributions $D_{K T}$ in $k_{T}$ factorization:

$$
\begin{aligned}
D_{K T}\left(x, q_{\perp}^{2}\right) \approx & \int_{x}^{1} \frac{\mathrm{d} \beta}{\beta} \int_{0}^{r} \frac{\mathrm{d} k_{\perp}^{2}}{k_{\perp}^{2}} D_{K T}^{(\text {pert })} \\
& \left(x / \beta, q_{\perp}^{2} / k_{\perp}^{2}\right) \Phi\left(w \beta, k_{\perp}^{2}\right),
\end{aligned}
$$

where $r=w \beta-q_{\perp}^{2}$. Obviously, $r \approx w \approx q_{\perp}^{2}$ for large $x$, while at very small $x$ one can use $r \approx w \beta$. $\Phi$ denotes the primary (i.e. non-perturbative) $k_{T}$-parton distribution. It is related to $\Psi$ as follows:

$\Phi\left(w \beta, k_{\perp}\right)=\int_{0}^{k_{\perp}^{2} / w \beta} \mathrm{d} \alpha \Psi\left(w \alpha, k_{\perp}^{2}\right)$.

\subsection{Reduction to collinear factorization}

In Ref. [23,24] we discussed how to reduce $K_{T}$-factorization to the collinear one, using DIS structure functions as an example. The same argumentation can be applied to the parton distributions. We briefly repeat it below. In order to reduce $k_{T^{-}}$ factorization to collinear factorization, we should perform the integration of Eq. (35) with respect to $k_{\perp}$ without integrating $D^{\text {(pert) }}$. Of course this cannot be done straightforwardly, because $D^{\text {(pert) }}$ explicitly depends on $k_{\perp}$. However, we can do it approximately, assuming a sharp peaked dependence of $\Psi\left(w \alpha, k_{\perp}^{2}\right)$ on $k_{\perp}^{2}$ with maximum at $k_{\perp}^{2}=\mu^{2}$, as shown in Fig. 7. The closer this dependence is to $\delta\left(k_{\perp}^{2}-\mu^{2}\right)$, the higher is the accuracy of the reduction. As discussed in Ref. [23,24], the number of such maximums can be unlimited. We recall that $\Phi$ is non-perturbative, so typical values of $\mu$ must be in the non-perturbative range, $\mu \sim \Lambda_{Q C D}$. After the integration of $\Phi$ we arrive at the collinear factorization convolution

$$
D^{(\mathrm{col})}\left(x, q_{\perp}^{2} / \mu^{2}\right) \approx \int_{x}^{1} \frac{\mathrm{d} \beta}{\beta} D_{c o l}^{(\mathrm{pert})}\left(x / \beta, q_{\perp}^{2} / \mu^{2}\right) \phi\left(\beta, \mu^{2}\right),
$$

with $\mu$ being the intrinsic factorization scale and $\phi$ being the primary (non-perturbative) integrated parton distribution:

$\phi\left(\beta, \mu^{2}\right)=\int_{\Omega} \frac{\mathrm{d} k_{\perp}^{2}}{k_{\perp}^{2}} \Phi\left(w \beta, k_{\perp}^{2}\right)$, 


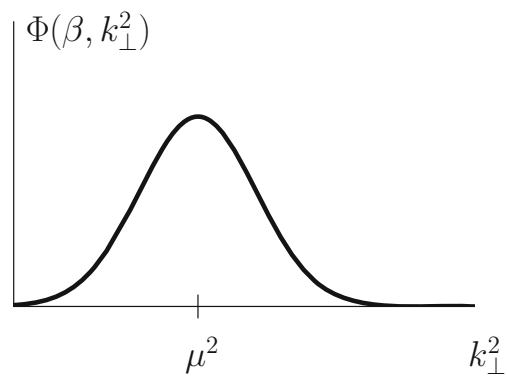

Fig. 7 The peaked form of $\Phi\left(\beta, k_{\perp}^{2}\right)$ with one maximum

where the integration region $\Omega$ is located around the maximum $k_{\perp}^{2}=\mu^{2}$. At first sight, the form of the collinear factorization presented in Eq. (37) contradicts the conventional form. Indeed, the scale $\mu$ in Eq. (37) corresponds to the maximum in Fig. 7 and therefore its value is fixed. On the contrary, the conventional form of the collinear factorization operates with integrated parton distributions $\varphi\left(\beta, \widetilde{\mu}^{2}\right)$, where the scale $\tilde{\mu}$ can have any arbitrary value. Then we expect the value of $\mu$ to be in the non-perturbative range, where, as usually, $\tilde{\mu} \sim$ few $\mathrm{GeV}$, i.e. typically $\tilde{\mu} \gg \mu$. However, this contradiction can easily be solved as was shown in Ref. [23,24]. The point is that a transition from $\phi\left(\beta, \mu^{2}\right)$ to $\varphi\left(\beta, \widetilde{\mu}^{2}\right)$ can be done, applying the perturbative evolution in the $\mu^{2}$-space to $\phi\left(\beta, \mu^{2}\right)$ and keeping $\beta$ fixed. It can be written symbolically as

$\varphi\left(\beta, \tilde{\mu}^{2}\right)=E\left(\tilde{\mu}^{2}, \mu^{2}\right) \otimes \phi\left(\beta, \mu^{2}\right)$,

where $E$ is the evolution operator in the $\mu^{2}$-space. Specific expressions for $E$ are different in different perturbative approaches (see Appendix E for details). Equation (39) makes it possible to arrive at the conventional unintegrated distribution $\varphi\left(\beta, \widetilde{\mu}^{2}\right)$, fixed at an arbitrary scale $\widetilde{\mu}^{2}$. In contrast to $\phi\left(\beta, \mu^{2}\right)$, the distribution $\varphi\left(\beta, \tilde{\mu}^{2}\right)$ accumulates both perturbative and non-perturbative contributions. It is easy to show that our reasoning remains true in the case when $\Phi$ has several maximums or an infinite series of them. This point was discussed in detail in Ref. [23,24], so we will not do it in the present paper. Instead, we focus on the modeling of invariant amplitudes $T^{(U, S)}$ as introduced in Eq. (25).

\section{Modeling the invariant quark-hadron amplitudes and primary quark distributions}

In this section we suggest a model which mimics nonperturbative QCD contributions in the primary hadron-quark invariant amplitudes $T^{(U, S)}$ and in the primary quark distributions in all available forms of factorization. Once again we begin with a consideration of the invariant amplitudes $T^{(U, S)}$ and then proceed to the quark distributions.
5.1 Resonance model for the primary quark-hadron invariant amplitudes

The amplitudes $T^{(U, S)}$ can be introduced in a modeldependent way only because QCD has not been solved in the non-perturbative region. All such models should satisfy several restrictions:

(i) The IR stability conditions in Eq. (12) and the UV stability conditions in Eqs. (20-23) should be respected, because they guarantee integrability of the factorization convolutions. We recall that the UV stability conditions derived in Sect. 2 depend on the UV-behavior of the factors regulating IR divergences. Namely, Eqs. (14), (20), and (22) correspond to the case (A), while Eqs. (15), (21), and (23) correspond to the case (B). In the present paper we focus on the most UVdivergent case (A), although our conclusions hold true for the case (B) as well.

(ii) The invariant amplitudes should respect the optical theorem, so they should have $s$-channel imaginary parts.

(iii) These amplitudes should guarantee the step-by-step reductions from basic factorization to $k_{T}$-factorization and then to collinear factorization as described in Sect. 4.

The expressions for the unpolarized and spin-dependent amplitudes with the longitudinal spin in Eq. (30) are much alike, while the expression for the transverse spin amplitude differs from them. Despite this difference, our model equally stands for all spin-dependent amplitudes and quark distributions, regardless of the spin orientation. To describe the invariant primary quark-hadron amplitudes, we suggest a model of the resonance type for $T_{\mathrm{q}}^{(U, S)}$ :

$$
\begin{aligned}
& T_{\mathrm{q}}^{(U)}\left(p k, k^{2}\right) \\
& =\frac{R_{U}\left(k^{2}\right)}{\left((k-p)^{2}-M_{1}^{2}+\imath \Gamma_{1}\right)\left((k-p)^{2}-M_{2}^{2}+\imath \Gamma_{2}\right)} \\
& T_{\mathrm{q}}^{(S)}\left(p k, k^{2}\right) \\
& =\frac{R_{S}\left(k^{2}\right)}{\left((k-p)^{2}-M_{3}^{2}+\imath \Gamma_{3}\right)\left((k-p)^{2}-M_{4}^{2}+\imath \Gamma_{4}\right)}
\end{aligned}
$$

where the $R_{U, S}\left(k^{2}\right)$ are supposed to behave as $R_{U, S}\left(k^{2}\right) \sim$ $\left(k^{2}\right)^{1+\eta}$ at small $k^{2}$. We need at least two resonances to satisfy the UV stability requirement of Eq. (14). Indeed, Eq. (40) leads to $\chi=1$, while a model with one resonance corresponds to $\chi=0$. Formally, Eq. (40) contains the independent parameters $M_{1,2,3,4}$ and $\Gamma_{1,2,3,4}$ but we do not see a physical reason forbidding us to identify $T_{\mathrm{q}}^{(U)}$ and $T_{\mathrm{q}}^{(S)}$, which would leave us with the parameters $M_{1,2}$ and $\Gamma_{1,2}$ only. In terms of the Sudakov variables, the $T_{\mathrm{q}}^{(U, S)}$ are

$$
\begin{aligned}
& T_{\mathrm{q}}^{(U)}\left(w \alpha, k^{2}\right) \\
& =\frac{R_{U}\left(k^{2}\right)}{\left(w \alpha+k^{2}-\mu_{1}^{2}+\imath \Gamma_{1}\right)\left(w \alpha+k^{2}-\mu_{2}^{2}+\imath \Gamma_{2}\right)}
\end{aligned}
$$




$$
\begin{aligned}
& T_{\mathrm{q}}^{(S)}\left(w \alpha, k^{2}\right) \\
& =\frac{R_{S}\left(k^{2}\right)}{\left(w \alpha+k^{2}-\mu_{3}^{2}+\imath \Gamma_{3}\right)\left(w \alpha+k^{2}-\mu_{4}^{2}+\imath \Gamma_{4}\right)},
\end{aligned}
$$

where $k^{2}=-w \alpha \beta-k_{\perp}^{2}$ and

$\mu_{j}^{2}=M_{j}^{2}-p^{2}$.

We suggest that the values of $\mu_{j}^{2}$ and $\Gamma_{j}$ should be within the non-perturbative scale domain, with $M_{j}^{2}>\Gamma_{j}$. It is convenient to write $T^{U, S}$ as the sum of two resonances:

$$
\begin{aligned}
T_{\mathrm{q}}^{(U)}\left(w \alpha, k^{2}\right) \\
=\frac{R_{U}\left(k^{2}\right)}{\left(\mu_{1}^{2}-\mu_{2}^{2}\right)-\imath\left(\Gamma_{1}-\Gamma_{2}\right)} \\
\quad \times\left[\frac{1}{\left(w \alpha+k^{2}-\mu_{1}^{2}+\imath \Gamma_{1}\right)}-\frac{1}{\left(w \alpha+k^{2}-\mu_{2}^{2}+\imath \Gamma_{2}\right)}\right], \\
=\frac{T_{\mathrm{q}}^{(S)}\left(w \alpha, k^{2}\right)}{\left(\mu_{3}^{2}-\mu_{4}^{2}\right)-\imath\left(\Gamma_{3}-\Gamma_{4}\right)} \\
\quad \times\left[\frac{1}{\left(w \alpha+k^{2}-\mu_{3}^{2}+\imath \Gamma_{3}\right)}-\frac{1}{\left(w \alpha+k^{2}-\mu_{4}^{2}+\imath \Gamma_{4}\right)}\right] .
\end{aligned}
$$

It seems that specifying $R_{U, S}$ cannot be done unambiguously. We postpone investigating this problem to the future, while in the present paper we use $R_{U, S}$ defined as follows:

$R_{U}=\lambda_{U}\left(\frac{k^{2}}{k^{2}+\mu_{U}^{2}}\right)^{1+\eta}, \quad R_{S}=\lambda_{S}\left(\frac{k^{2}}{k^{2}+\mu_{S}^{2}}\right)^{1+\eta}$

where $\lambda_{U, S}$ and $\mu_{U, S}^{2},\left(\mu_{U, S}^{2}>0\right)$ are independent parameters, though we think that $R_{U}$ and $R_{S}$ could coincide, which would diminish the number of free parameters. It is easy to check now that the expressions for $T_{\mathrm{q}}^{(U)}, T_{\mathrm{q}}^{(S)}$ introduced in Eq. (41) obey the condition of IR stability in Eq. (12) with arbitrary $\eta$. In contrast, the value of the UV parameter $\chi$ (introduced in Eqs. (14) and (20) to guarantee UV stability) is now fixed: $\chi=1$ in Eq. (41). Equations (25) and (41) are suggested for invariant amplitudes $T_{\mathrm{q}}^{(U, S)}$ in basic factorization. Reducing basic factorization to $k_{T}$-factorization converts $T_{\mathrm{q}}^{(U, S)}$ into new amplitudes $\widetilde{T}_{\mathrm{q}}^{(U, S)}$. They are obtained from $T_{\mathrm{q}}^{(U, S)}$ by integrating them with respect to $\alpha$ :

$\widetilde{T}_{\mathrm{q}}^{(r)}\left(\beta, k_{\perp}^{2}\right)=\int_{0}^{\alpha_{\max }} \mathrm{d} \alpha T^{(r)}\left(\alpha, k^{2}\right)$,

where $r=U, S$. The upper limit of integration, $\alpha_{\max }$ should obey Eq. (34), so we choose

$\alpha_{\max } \approx k_{\perp}^{2} /(w \beta)$.
According to Eq. (34), $k^{2} \approx-k_{\perp}^{2}$. The integration leads to the following expression for $\widetilde{T}_{\mathrm{q}}^{(j)}$ (see Appendix D for details):

$$
\begin{aligned}
& \widetilde{T}_{\mathrm{q}}^{(U)}\left(\beta, k_{\perp}^{2}\right) \approx \frac{1}{2} R_{U}\left(k_{\perp}^{2}\right) \\
& \quad \times\left[\frac{1}{k_{\perp}^{2}(1-\beta) / \beta-\mu_{1}^{2}+\imath \Gamma_{1}}+\frac{1}{k_{\perp}^{2}(1-\beta) / \beta-\mu_{2}^{2}+\imath \Gamma_{2}}\right] \\
& \quad+\Delta \widetilde{T}_{\mathrm{q}}^{(U)}, \\
& \widetilde{T}_{\mathrm{q}}^{(S)}\left(\beta, k_{\perp}^{2}\right) \approx \frac{1}{2} R_{S}\left(k_{\perp}^{2}\right) \\
& \quad \times\left[\frac{1}{k_{\perp}^{2}(1-\beta) / \beta-\mu_{3}^{2}+l \Gamma_{3}}+\frac{1}{k_{\perp}^{2}(1-\beta) / \beta-\mu_{4}^{2}+\imath \Gamma_{4}}\right] \\
& \quad+\Delta \widetilde{T}_{\mathrm{q}}^{(S)},
\end{aligned}
$$

where $\Delta \widetilde{T}_{\mathrm{q}}^{(U)}$ and $\Delta \widetilde{T}_{\mathrm{q}}^{(S)}$ are

$$
\begin{aligned}
& \Delta \widetilde{T}_{\mathrm{q}}^{(U)}=\frac{1}{\left(\mu_{1}^{2}-\mu_{2}^{2}\right)+\imath\left(\Gamma_{1}-\Gamma_{2}\right)} \ln \left(\frac{k_{\perp}^{2}+\mu_{1}^{2}-\imath \Gamma_{1}}{k_{\perp}^{2}+\mu_{2}^{2}-\imath \Gamma_{2}}\right), \\
& \Delta \widetilde{T}_{\mathrm{q}}^{(S)}=\frac{1}{\left(\mu_{3}^{2}-\mu_{4}^{2}\right)+\imath\left(\Gamma_{3}-\Gamma_{4}\right)} \ln \left(\frac{k_{\perp}^{2}+\mu_{3}^{2}-\imath \Gamma_{3}}{k_{\perp}^{2}+\mu_{4}^{2}-\imath \Gamma_{4}}\right) .
\end{aligned}
$$

They depend on $k_{\perp}$ very slowly and they can be neglected at large $k_{\perp}^{2}$.

\subsection{Primary quark distributions}

The optical theorem relates the $s$-channel imaginary parts of $T^{(U, S)}$ and $\widetilde{T}^{(U, S)}$ to the primary quark distributions $\Psi_{U, S}$ in basic factorization and to unintegrated (or) quark distributions $\Phi_{U, S}$ in $k_{T}$-factorization, respectively. So, applying the optical theorem, we obtain the following expression for the primary quark distribution $\Psi_{r}$ in basic factorization:

$$
\begin{aligned}
& \Psi_{U}\left(w \alpha, k^{2}\right)=\frac{1}{\pi} \frac{R_{U}\left(k^{2}\right)}{\left(\mu_{1}^{2}-\mu_{2}^{2}\right)} \\
& \times\left[\frac{\Gamma_{1}}{\left(w \alpha+k^{2}-\mu_{1}^{2}\right)^{2}+\Gamma_{1}^{2}}-\frac{\Gamma_{2}}{\left(w \alpha+k^{2}-\mu_{2}^{2}\right)^{2}+\Gamma_{2}^{2}}\right], \\
& \left.\Psi_{S}\left(w \alpha, k^{2}\right)=\frac{1}{\pi} \frac{R_{S}\left(k^{2}\right)}{\left(\mu_{3}^{2}-\mu_{4}^{2}\right)}\right] \\
& \quad \times\left[\frac{\Gamma_{3}}{\left(w \alpha+k^{2}-\mu_{3}^{2}\right)^{2}+\Gamma_{3}^{2}}-\frac{\Gamma_{4}}{\left(w \alpha+k^{2}-\mu_{4}^{2}\right)^{2}+\Gamma_{4}^{2}}\right],
\end{aligned}
$$

and a similar expression for the primary quark distribution $\Phi_{U, S}$ in $k_{T}$-factorization:

$$
\begin{aligned}
& \Phi_{U}\left(\beta, k_{\perp}^{2}\right)=\frac{1}{\pi} R_{U}\left(k_{\perp}^{2}\right) \\
& \quad \times\left[\frac{\Gamma_{1}}{\left(k_{\perp}^{2}(1-\beta) / \beta-\mu_{1}^{2}\right)^{2}+\Gamma_{1}^{2}}+\frac{\Gamma_{2}}{\left(k_{\perp}^{2}(1-\beta) / \beta-\mu_{2}^{2}\right)^{2}+\Gamma_{2}^{2}}\right],
\end{aligned}
$$




$$
\begin{aligned}
& \Phi_{S}\left(\beta, k_{\perp}^{2}\right)=\frac{1}{\pi} R_{S}\left(k_{\perp}^{2}\right) \\
& \quad \times\left[\frac{\Gamma_{3}}{\left(k_{\perp}^{2}(1-\beta) / \beta-\mu_{3}^{2}\right)^{2}+\Gamma_{3}^{2}}+\frac{\Gamma_{4}}{\left(k_{\perp}^{2}(1-\beta) / \beta-\mu_{4}^{2}\right)^{2}+\Gamma_{4}^{2}}\right] .
\end{aligned}
$$

Obviously, the expressions in Eqs. (49) and (50) are of the Breit-Wigner type. Substituting Eq. (50) in Eq. (35) and integrating over $k_{\perp}^{2}$, we arrive at the quark parton distribution $D_{j}^{(\mathrm{col})}$ in $k_{T}$-factorization, where the non-perturbative contributions i.e. the unintegrated parton distributions are specified:

$$
\begin{aligned}
& D_{U}^{\left(k_{T}\right)}\left(x, q_{\perp}^{2}\right)=\frac{1}{\pi} \int_{x}^{1} \frac{\mathrm{d} \beta}{\beta} \int_{0}^{r} \frac{\mathrm{d} k_{\perp}^{2}}{k_{\perp}^{2}} D_{U}^{\text {(pert })} \\
& \quad \times\left(x / \beta, k_{\perp}^{2} / q_{\perp}^{2}\right) R_{U}\left(k_{\perp}^{2}\right) \\
& \quad \times\left[\frac{\Gamma_{1}}{\left(k_{\perp}^{2}(1-\beta) / \beta-\mu_{1}^{2}\right)^{2}+\Gamma_{1}^{2}}\right. \\
& \left.\quad+\frac{\Gamma_{2}}{\left(k_{\perp}^{2}(1-\beta) / \beta-\mu_{2}^{2}\right)^{2}+\Gamma_{2}^{2}}\right] \\
& D_{S}^{\left(k_{T}\right)}\left(x, q_{\perp}^{2}\right)=\frac{1}{\pi} \int_{x}^{1} \frac{\mathrm{d} \beta}{\beta} \int_{0}^{r} \frac{\mathrm{d} k_{\perp}^{2}}{k_{\perp}^{2}} D_{S}^{(\mathrm{pert})}\left(x / \beta, k_{\perp}^{2} / q_{\perp}^{2}\right) \\
& R_{S}\left(k_{\perp}^{2}\right)\left[\frac{\Gamma_{3}}{\left(k_{\perp}^{2}(1-\beta) / \beta-\mu_{3}^{2}\right)^{2}+\Gamma_{3}^{2}}\right. \\
& \left.+\frac{\Gamma_{4}}{\left(k_{\perp}^{2}(1-\beta) / \beta-\mu_{4}^{2}\right)^{2}+\Gamma_{4}^{2}}\right]
\end{aligned}
$$

where $r$ is defined in Eq. (35). Let us consider the $k_{\perp}$ dependence in Eqs. (35) and (50) in more detail. Obviously, the structures of the expressions for $D_{U}^{\left(k_{T}\right)}$ and $D_{S}^{\left(k_{T}\right)}$ (or $\Phi_{u}$ and $\left.\Phi_{S}\right)$ are quite similar, so we consider $D_{U}^{\left(k_{T}\right)}$ only. Then, the expression in the squared brackets in Eq. (35), i.e. $\Phi_{U}$ of Eq. (50), is symmetric with respect to the replacement $1 \rightleftharpoons 2$. Each term in the parentheses has a peaked form, with maximums at $k_{\perp}^{2}=\mu_{1,2}^{2}$. The smaller $\Gamma_{1,2}$ is, the sharper the peaks are. We recall that $R_{U, S} \sim\left(k_{\perp}^{2}\right)^{1+\eta}$ at small $k_{\perp}^{2}$. By definition, see Eq. (42), $\mu_{1,2}^{2}=M_{1,2}^{2}-p^{2}$, so they can be either positive or negative, while $k_{\perp}^{2}$ cannot be negative. In any case, both terms in $\Phi_{U}$ and $\Phi_{S}$ contribute to $D_{U, S}^{\left(k_{T}\right)}$, but the result of interference of the two peaks depends on the values of the parameters. There are three particular cases possible.

Case (i) both $\mu_{1}^{2}$ and $\mu_{2}^{2}$ are positive.

In this case both maximums are within the integration region of Eq. (35) and interference of the two peaks generates various forms of $\Phi_{U}\left(\beta, k_{\perp}^{2}\right)$, ranging from the picture with two isolated peaks to a kind of plateau, depending on the values of $\Gamma_{1,2}$.

Case (ii) $\mu_{1}^{2}>0$ and $\mu_{2}^{2}<0$ or vice versa.
Here the peak from the first term in Eq. (50) combines with a tail of the contribution of the second term whose maximum is beyond the integration region of Eq. (35). The resulting picture has a resemblance to the dual model combining a resonant and a constant term.

\section{Case (iii) both $\mu_{1}^{2}$ and $\mu_{2}^{2}$ are negative.}

The two maximums now are out of the integration region, so tails of the peaks, taken by themselves, generate a form slowly decreasing with the growth of $k_{\perp}^{2}$. However, this slope is affected by the impact of $R_{U}$. We recall that $R_{U}=0$ at $k_{\perp}^{2}=0$.

\subsection{Primary quark distributions in collinear factorization}

Performing an integration over $k_{\perp}^{2}$ in Eq. (35), we arrive at the parton distributions $D_{j}^{(\mathrm{col})}$ in collinear factorization. Presuming that the parameter $\Gamma_{j}$ is small, we write the result of the integration in the following form (see Appendix $\mathrm{C}$ for details):

$$
\begin{aligned}
& D_{U}^{(\mathrm{col})}\left(x, q_{\perp}^{2}\right) \approx \int_{x}^{1} \frac{\mathrm{d} \beta}{\beta} D_{U}^{(\mathrm{pert})}\left(x / \beta, q_{\perp}^{2} / \mu_{1}^{2}\right) \phi_{U}\left(\beta, \mu_{1}^{2}\right) \\
& +\int_{x}^{1} \frac{\mathrm{d} \beta}{\beta} D_{U}^{\text {(pert) }}\left(x / \beta, q_{\perp}^{2} / \mu_{2}^{2}\right) \phi_{U}\left(\beta, \mu_{2}^{2}\right),
\end{aligned}
$$

with

$\phi_{U}\left(\beta, \mu_{1}^{2}\right) \approx \frac{1}{\pi} \int_{\Omega_{1}} \frac{\mathrm{d} k_{\perp}^{2}}{k_{\perp}^{2}} \frac{R_{U}\left(k_{\perp}^{2}\right) \Gamma_{1}}{\left(k_{\perp}^{2}(1-\beta) / \beta-\mu_{1}^{2}\right)^{2}+\Gamma_{1}^{2}}$,
$\phi_{U}\left(\beta, \mu_{2}^{2}\right) \approx \frac{1}{\pi} \int_{\Omega_{2}} \frac{\mathrm{d} k_{\perp}^{2}}{k_{\perp}^{2}} \frac{R_{U}\left(k_{\perp}^{2}\right) \Gamma_{2}}{\left(k_{\perp}^{2}(1-\beta) / \beta-\mu_{2}^{2}\right)^{2}+\Gamma_{2}^{2}}$,

where the integration regions are $\Omega_{1}=\Omega_{1}^{\prime} \bigcap[0, w]$ and $\Omega_{1}=\Omega_{2}^{\prime} \bigcap[0, w]$, with the subregions $\Omega_{1}^{\prime}, \Omega_{2}^{\prime}$ being located around the maximums of the peaks. Formally, both terms in Eq. (53) contribute to $\phi_{U}$ at any signs of $\mu_{1}^{2}, \mu_{2}^{2}$, but in the limit of sharp peaks these contributions have different weights. At $\mu_{1}^{2}>0, \mu_{2}^{2}>0$ the two terms contribute equally:

$$
\begin{aligned}
\phi_{U} \approx & R_{U}\left(\mu_{1}^{2} \beta /(1-\beta)\right) / \mu_{1}^{2}+R_{U}\left(\mu_{2}^{2} \beta /(1-\beta)\right) / \mu_{2}^{2} \\
& +O\left(\Gamma_{1}, \Gamma_{2}\right) .
\end{aligned}
$$

Mostly the first term contributes, when $\mu_{1}^{2}>0, \mu_{2}^{2}<0$ :

$\phi_{U} \approx R_{U}\left(\mu_{1}^{2} \beta /(1-\beta)\right) / \mu_{1}^{2}+O\left(\Gamma_{1}\right)$,

and vice versa. Finally, at $\mu_{1}^{2}, \mu_{2}^{2}<0$ only the tails of the two peaks contribute and therefore $\phi_{U}$ is small and flat compared to the previous cases:

$\phi_{U} \approx$ const. 
(see Appendix E for details). When $\mu_{1}^{2}>0, \mu_{2}^{2}>0$

$$
\begin{aligned}
\varphi_{U}\left(\omega, \mu^{2}\right)= & \int_{x}^{1} \frac{\mathrm{d} \beta}{\beta} \beta^{\omega}\left[E\left(\omega, \mu^{2}, \mu_{1}^{2}\right) R_{U}\left(\mu_{1}^{2} \beta /(1-\beta)\right) \mu_{2}^{-2}\right. \\
& \left.+E\left(\omega, \mu^{2}, \mu_{2}^{2}\right) R_{U}\left(\mu_{2}^{2} \beta /(1-\beta)\right) \mu_{2}^{-2}\right]
\end{aligned}
$$

$$
R_{U}\left(\mu_{j}^{2} \beta /(1-\beta)\right) \mu_{j}^{-2}=\lambda_{U} \beta .
$$

Combining Eqs. (57) and (58), integrating over $\beta$ and remembering that at small $x$ the essential values of $\omega$ are small leads to the following expression for $\varphi_{U}\left(\omega, \mu^{2}\right)$ (see Appendix E for details):

$$
\begin{aligned}
\varphi_{U}\left(\omega, \mu^{2}\right)= & \int_{x}^{1} \frac{\mathrm{d} \beta}{\beta} \beta^{\omega+1}\left[\frac{\lambda_{U}}{\mu_{1}^{2}} E\left(\omega, \mu^{2}, \mu_{1}^{2}\right)\right. \\
& \left.+\frac{\lambda_{U}}{\mu_{1}^{2}} E\left(\omega, \mu^{2}, \mu_{2}^{2}\right)\right] \\
\approx & \frac{\lambda_{U}}{\mu_{1}^{2}} E\left(\omega, \mu^{2}, \mu_{1}^{2}\right)+\frac{\lambda_{U}}{\mu_{2}^{2}} E\left(\omega, \mu^{2}, \mu_{2}^{2}\right) .
\end{aligned}
$$

\section{Conclusion}

In the present paper we have considered the quark-hadron scattering amplitudes and distributions of polarized and unpolarized quarks in hadrons in the framework of the factorization concept where both amplitudes and distributions are expressed through convolutions of the perturbative and non-perturbative components. We began with considering the quark-hadron amplitudes in basic factorization where the integration over the momenta of the connecting partons runs over the whole phase space, and we obtained the conditions for the factorization convolution to be stable both in the IR and the UV regions. Then we demonstrated how to reduce basic factorization to $K_{T}$ - and collinear factorizations. We suggested a resonance model for non-perturbative contributions to the unpolarized and spin-dependent parton-hadron scattering amplitudes. This model is based on the following simple argumentation: after emitting an active quark by a hadron, the remaining colored quark-gluon state cannot be stable and therefore it can be described by quasi-resonant expressions. We needed at least two resonances in basic factorization and this remained true when basic factorization was reduced to $K_{T}$-factorization. Applying the optical theorem to the resonance model provided us first with the expressions of the Breit-Wigner type for non-perturbative (primary) contributions to the quark distributions in the basic and $K_{T^{-}}$ factorizations and then, after one more reduction, to the parton distributions in collinear factorization. To conclude, let us notice that the resonance model can also be used for the analysis of the non-singlet components of the DIS structure functions.
Acknowledgments We are grateful to B.L. Webber for interesting discussions. S.I. Troyan is supported by the Russian Science Foundation, Grant No. 14-22-00281.

Open Access This article is distributed under the terms of the Creative Commons Attribution 4.0 International License (http://creativecomm ons.org/licenses/by/4.0/), which permits unrestricted use, distribution, and reproduction in any medium, provided you give appropriate credit to the original author(s) and the source, provide a link to the Creative Commons license, and indicate if changes were made.

Funded by $\mathrm{SCOAP}^{3}$.

\section{Appendix A: Amplitude for the forward Compton scattering off a gluon in the box approximation}

We have

$$
\begin{aligned}
M_{\mu \nu \lambda \rho}= & \left(t_{l} t_{r}\right) \frac{\mathrm{e}^{2} \alpha_{s}}{8 \pi^{2}} w \int \mathrm{d} \beta^{\prime} \mathrm{d} \alpha^{\prime} \mathrm{d} k_{\perp}^{\prime 2} \\
& \times\left[M_{\mu \nu \lambda \rho}^{(1)}+M_{\mu \nu \lambda \rho}^{(2)}+M_{\mu \nu \lambda \rho}^{(3)}\right] \\
M_{\mu \nu \lambda \rho}^{(1)}= & \frac{\operatorname{Tr}\left[\gamma_{\nu}\left(\hat{q}+\hat{k}^{\prime}\right) \gamma_{\mu} \hat{k}^{\prime} \gamma_{\lambda}\left(\hat{k^{\prime}}-\hat{k}\right) \gamma_{\rho} \hat{k}^{\prime}\right]}{k^{\prime 2} k^{\prime 2}\left(q+k^{\prime}\right)^{2}\left(k^{\prime}-k\right)^{2}}, \\
M_{\mu \nu \lambda \rho}^{(2)}= & \frac{\operatorname{Tr}\left[\gamma_{\nu}\left(\hat{q}+\hat{k}^{\prime}\right) \gamma_{\mu} \hat{k}^{\prime} \gamma_{\rho}\left(\hat{k^{\prime}}+\hat{k}\right) \gamma_{\lambda} \hat{k^{\prime}}\right]}{k^{\prime 2} k^{\prime 2}\left(q+k^{\prime}\right)^{2}\left(k^{\prime}+k\right)^{2}}, \\
M_{\mu \nu \lambda \rho}^{(3)}= & \frac{\operatorname{Tr}\left[\gamma_{\nu}\left(\hat{q}+\hat{k}^{\prime}-\hat{k}\right) \gamma_{\rho}\left(\hat{k}^{\prime}-\hat{k}\right) \gamma_{\mu} \hat{k}^{\prime} \gamma_{\lambda}\left(\hat{k^{\prime}}+\hat{q}\right)\right]}{k^{\prime 2}\left(q+k^{\prime}\right)^{2}\left(k^{\prime}-k\right)^{2}\left(q+k^{\prime}-k\right)^{2}} .
\end{aligned}
$$

\section{Appendix B: Projection operators for forward Compton amplitudes}

The conventional way of dealing with the forward Compton scattering amplitude $A_{\mu v}$ is, in the first place, to simplify their tensor structure. To this end, $A_{\mu \nu}$ is represented as an expansion of $A_{\mu \nu}$ into a series of simpler tensors, each multiplied by an invariant amplitude. Such tensors are called projection operators. Through the optical theorem the invariant amplitudes are related to the DIS structure functions.

In the case of the unpolarized Compton scattering such an expansion looks as follows:

$A_{\mu \nu}=P_{\mu \nu}^{(1)} A_{1}+P_{\mu \nu}^{(2)} A_{2}$,

where

$$
\begin{array}{r}
P_{\mu \nu}^{(1)}=-g_{\mu \nu}+q_{\mu} q_{\nu} / q^{2}, \quad P_{\mu \nu}^{(2)}=(1 / p q) \\
\left(p_{\mu}-q_{\mu}\left(p q / q^{2}\right)\right)\left(p_{\nu}-q_{\nu}\left(p q / q^{2}\right)\right)
\end{array}
$$

are the projection operators and $A_{1}, A_{2}$ are invariant amplitudes. According to the optical theorem

$F_{1}=\frac{1}{\pi} \Im A_{1}, \quad F_{2}=\frac{1}{\pi} \Im A_{2}$. 
Similarly, for the polarized Compton scattering

$A_{\mu \nu}=P_{\mu \nu}^{(3)} A_{3}+P_{\mu \nu}^{(4)} A_{4}$,

where

$$
\begin{aligned}
P_{\mu \nu}^{(3)} & =\imath \epsilon_{\mu \nu \lambda \rho} M q_{\lambda} S_{\rho}, P_{\mu \nu}^{(4)} \\
& =\imath \epsilon_{\mu \nu \lambda \rho} M q_{\lambda}\left[S_{\rho}-p_{\rho}(q S / q p)\right],
\end{aligned}
$$

with $M$ and $S$ being the hadron mass and spin, respectively, and $A_{3,4}$ are spin-dependent invariant amplitudes. The optical theorem states that

$g_{1}=\frac{1}{\pi} \Im A_{3}, g_{2}=\frac{1}{\pi} \Im A_{4}$.

All operators $P_{\mu \nu}^{(n)}$ respect the electromagnetic current conservation: $q_{\mu} P_{\mu \nu}^{(n)}=q_{\nu} P_{\mu \nu}^{(n)}=0$. It is convenient to introduce the longitudinal, $S^{\|}$, and transverse, $S^{\perp}$, components of the spin, so that $S^{\perp} p=S^{\perp} q=0$ and $S_{\rho}^{\|}=p_{\rho}(q S / p q)$. In such terms Eq. (B4) can be written as follows:

$$
\begin{aligned}
A_{\mu \nu} & =\imath \epsilon_{\mu \nu \lambda \rho} M q_{\lambda}\left[S_{\rho}^{\|} A_{3}+S_{\rho}^{\perp}\left(A_{3}+A_{4}\right)\right] \\
& \equiv \iota \epsilon_{\mu \nu \lambda \rho} M q_{\lambda}\left[S_{\rho}^{\|} A^{\|}+S_{\rho}^{\perp} A^{\perp}\right] .
\end{aligned}
$$

This expression is useful for practical attributing different terms in the spin-dependent $A_{\mu \nu}$ to the proper invariant amplitudes. In the unpolarized case one can use the simple rule: expressions $\sim g_{\mu \nu}$ contribute to $A_{1}$, while expressions $\sim p_{\mu} p_{\nu} / p q$ form $A_{2}$. In contrast, the gauge invariance admits adding arbitrary terms $\sim q_{\mu}, q_{\nu}$.

\section{Appendix C: Convolutions involving the Breit-Wigner formula}

Let us consider the following convolution:

$F=\frac{1}{\pi} \int_{-\infty}^{\infty} \mathrm{d} x f(x) \frac{\Gamma}{\left(x-x_{0}\right)^{2}+\Gamma^{2}}$.

Replacing $x$ by $t$, with $t=\left(x-x_{0}\right) / \Gamma$, we convert Eq. (C1) into

$F=\frac{1}{\pi} \int_{-\infty}^{\infty} d t f\left(t \Gamma+x_{0}\right) \frac{\Gamma}{t^{2}+1}$.

At small $\Gamma$, we can expand $f\left(t \Gamma+x_{0}\right)$ in the power series and retain several terms:

$f\left(t \Gamma+x_{0}\right)=f\left(x_{0}\right)+f^{\prime}\left(x_{0}\right) t \Gamma+O\left(\Gamma^{2}\right)$.

Substituting Eq. (C3) in (C2) and integrating (C2) yields

$F=f\left(x_{0}\right)+O(\Gamma)$.

The first term in Eq. (C4) corresponds to the well-known representation of the $\delta$-function:

$\frac{1}{\pi} \lim _{\epsilon \rightarrow 0} \frac{\epsilon}{x^{2}+\epsilon^{2}}=\delta(x)$.
Appendix D: Integration in Eq. (45)

A generic expression to integrate can be written as

$$
\begin{aligned}
\widetilde{T}= & \int_{0}^{\alpha_{\max }} \frac{\mathrm{d} \alpha}{(\alpha-A)(\alpha-B)}=\frac{1}{(A-B)} \\
& \times \int_{0}^{\alpha_{\max }} \mathrm{d} \alpha\left[\frac{1}{\alpha-A}-\frac{1}{\alpha-B}\right]=\frac{1}{(A-B)} \\
& \times\left[\ln \left(\frac{\alpha_{\max }-A}{\alpha_{\max }-B}\right)-\ln \left(\frac{A}{B}\right)\right] .
\end{aligned}
$$

Assuming that $\alpha_{\max } \gg A, B \gg|A-B|$ allows us to expand the logarithms into the power series and retain the first terms only, we have

$\widetilde{T} \approx \frac{1}{2}\left[\frac{1}{\alpha_{\max }-A}+\frac{1}{\alpha_{\max }-B}\right]-\frac{1}{(A-B)} \ln (A / B)$.

We have written Eq. (D2) in the symmetrical form with respect to $A, B$ because Eq. (D1) has this feature.

\section{Appendix E: Evolving the factorization scale in collinear factorization}

Using the Mellin transform, we can rewrite Eq. (37) as follows:

$$
\begin{aligned}
D^{(\mathrm{col})}\left(x, q_{\perp}^{2}\right)= & \int_{-l \infty}^{l \infty} \frac{\mathrm{d} \omega}{2 \pi \imath} x^{-\omega} C_{U}(\omega) E\left(\omega, q^{2}, \mu^{2}\right) \\
& \times \phi\left(\omega, \mu^{2}\right),
\end{aligned}
$$

where the primary quark distribution is fixed at the scale $\mu$, with $\mu^{2}<q_{\perp}^{2} . E\left(\omega, q_{\perp}^{2}, \mu^{2}\right)$ is a generic notation for an operator evolving the distribution $\phi\left(\omega, \mu^{2}\right)$ from the factorization scale $\mu^{2}$ to $q_{\perp}^{2}$, while $C(\omega)$ is responsible for the $x$ -evolution. Choosing a scale $\tilde{\mu}$ such that $\mu^{2}<\tilde{\mu}<q_{\perp}^{2}$ and representing $E\left(\omega, q_{\perp}^{2}, \mu^{2}\right)$ as

$E\left(\omega, q_{\perp}^{2}, \mu^{2}\right)=E\left(\omega, q_{\perp}^{2}, \tilde{\mu}^{2}\right) E\left(\omega, \tilde{\mu}^{2}, \mu^{2}\right)$,

we bring $D^{(\mathrm{col})}$ to the conventional form

$$
\begin{aligned}
D^{(\mathrm{col})}\left(x, q_{\perp}^{2}\right)= & \int_{-l \infty}^{l \infty} \frac{\mathrm{d} \omega}{2 \pi \imath} x^{-\omega} C_{U}(\omega) E\left(\omega, q^{2}, \tilde{\mu}^{2}\right) \\
& \times \varphi\left(\omega, \tilde{\mu}^{2}\right),
\end{aligned}
$$

where

$\varphi\left(\omega, \tilde{\mu}^{2}\right)=E\left(\omega, \tilde{\mu}^{2}, \mu^{2}\right) \phi\left(\beta, \mu^{2}\right)$,

which corresponds to Eq. (39). Actually, $\varphi\left(\omega, \tilde{\mu}^{2}\right)$ is the conventional parton distribution in the $\omega$-space (momentum space). It is fixed at an arbitrary scale $\widetilde{\mu}^{2}$ and related to the standard integrated distribution $\delta q\left(x, \widetilde{\mu}^{2}\right)$ by the Mellin transform. The evolution operator $E\left(\omega, q^{2}, \mu^{2}\right)$ is expressed 
in different terms, depending on the perturbative approach in use. For instance, in LO DGLAP with fixed $\alpha_{s}$ it is given by

$E=\exp \left[\alpha_{s} \gamma_{0}(\omega) \ln \left(q^{2} / \mu^{2}\right)\right]$

with $\gamma_{0}$ being the LO DGLAP anomalous dimension and $C_{F}=4 / 3$. When in LO DGLAP $\alpha_{s}$ is running and the standard parametrization $\alpha_{s}=\alpha_{s}\left(k_{\perp}^{2}\right)$ is used in the Feynman graphs, Eq. (E5) is changed,

$E=\left(\frac{q^{2}}{\mu^{2}}\right)^{\gamma_{0} / b}$,

with $b$ being the first coefficient of the $\beta$-function. The parametrization $\alpha_{s}=\alpha_{s}\left(k_{\perp}^{2}\right)$ should not be used at small $x$ (see Ref. [36]). When it is replaced by the appropriate parametrization and when the total resummation of the leading logarithms is done, Eq. (E6) is replaced by

$E=\exp \left[h(\omega) \ln \left(q^{2} / \mu^{2}\right)\right]$

where $h(\omega)$ is a new anomalous dimension. It accounts for the total resummation of the leading double-logarithmic contributions and running QCD coupling effects (see Ref. [34,35] and the overview [36] for details).

\section{References}

1. D. Amati, R. Petronzio, G. Veneziano, Nucl. Phys. B 140, 54 (1978)

2. A.V. Efremov, A.V. Radyushkin, Teor. Mat. Fiz. 42, 147 (1980)

3. A.V. Efremov, A.V. Radyushkin, Theor. Math. Phys. 44, 573 (1980)

4. A.V. Efremov, A.V. Radyushkin, Teor. Mat. Fiz. 44, 17 (1980)

5. A.V. Efremov, A.V. Radyushkin, Phys. Lett. B 63, 449 (1976)

6. A.V. Efremov, A.V. Radyushkin, Lett. Nuovo Cim. 19, 83 (1977)

7. S. Libby, G. Sterman, Phys. Rev. D 18, 3252 (1978)

8. S.J. Brodsky, G.P. Lepage, Phys. Lett. B 87, 359 (1979)

9. S.J. Brodsky, G.P. Lepage, Phys. Rev. D 22, 2157 (1980)
10. J.C. Collins, D.E. Soper, Nucl. Phys. B 193, 381 (1981)

11. J.C. Collins, D.E. Soper, Nucl. Phys. B 194, 445 (1982)

12. J.C. Collins, D.E. Soper, G. Sterman, Nucl. Phys. B 250, 199 (1985)

13. A.V. Efremov, A.V. Radyushkin, Report JINR E2-80-521. Mod. Phys. Lett. A 24, 2803 (2009)

14. G. Altarelli, G. Parisi, Nucl. Phys. B 126, 297 (1977)

15. V.N. Gribov, L.N. Lipatov, Sov. J. Nucl. Phys. 15, 438 (1972)

16. L.N. Lipatov, Sov. J. Nucl. Phys. 20, 95 (1972)

17. YuL Dokshitzer, Sov. Phys. JETP 46, 641 (1977)

18. E.A. Kuraev, L.N. Lipatov, V.S. Fadin, Sov. Phys. JETP 44, 443 (1976)

19. E.A. Kuraev, L.N. Lipatov, V.S. Fadin, Sov. Phys. JETP 45, 199 (1977)

20. I.I. Balitsky, L.N. Lipatov, Sov. J. Nucl. Phys. 28, 822 (1978)

21. S. Catani, M. Ciafaloni, F. Hautmann, Phys. Lett. B 242, 97 (1990)

22. S. Catani, M. Ciafaloni, F. Hautmann, Nucl. Phys. B 366, 135 (1991)

23. B.I. Ermolaev, M. Greco, S.I. Troyan, Eur. Phys. J. C 71, 1750 (2011)

24. B.I. Ermolaev, M. Greco, S.I. Troyan, Eur. Phys. J. C 72, 1953 (2012)

25. V.V. Sudakov, Sov. Phys. JETP 3, 65 (1956)

26. S.J. Brodsky, P. Hoyer, C. Peterson, N. Sakai, Phys. Lett. B 93, 451 (1980)

27. S.J. Brodsky, C. Peterson, N. Sakai, Phys. Rev. D 23, 2745 (1981)

28. J.C. Collins. PoS LC 2008, 028 (2008)

29. I.O. Cherednikov, N.G. Stefanis, AIP Conf. Proc. 1105, 327 (2009)

30. I.O. Cherednikov, N.G. Stefanis, Int. J. Mod. Phys. Conf. Ser. 4, 135-145 (2011)

31. O. Erdogan, G. Sterman, Phys. Rev. D 91(6), 065033 (2015)

32. J.C. Collins, T.C. Rogers, A.M. Stasto, Phys. Rev. D 77, 085009 (2008)

33. J. Collins, Int. J. Mod. Phys. Conf. Ser. 4, 85 (2011)

34. B.I. Ermolaev, M. Greco, S.I. Troyan, Nucl. Phys. B 571, 137 (2000)

35. B.I. Ermolaev, M. Greco, S.I. Troyan, Nucl. Phys. B 594, 71 (2001)

36. B.I. Ermolaev, M. Greco, S.I. Troyan, Riv. Nuovo Cimento 33, 37 (2010) 